\title{
Membrane Protein Structure Determination. Methods and Protocols
}

\author{
(J.-J. Lacapere (ed.) in Series "Springer Protocols. Methods in Molecular Biology" \\ (J. Walker, ser. ed.) Vol. 654, Humana Press, 2010, 459 p., \$139)
}

DOI: $10.1134 / \mathrm{S} 0006297911110113$

This book includes descriptions of modern methods employed for studies of membrane proteins, which represent nearly $40 \%$ of all proteins. The functional importance of these proteins cannot be overestimated. These proteins act as cell receptors, enzymes, and molecules triggering various metabolic processes. Membrane proteins are involved in intercellular interactions, and they also determine antigenic properties and many other biological processes. The study of membrane proteins is the most difficult part of proteomics, and it requires the use of complex special methods.

The book consists of five sections that include 23 chapters written by a representative international group of authors. Section I of this book deals with problems of membrane protein purification. Four chapters of this section (chapters 1-4) describe methods employed for preparation and purification of various natural and overexpressed membrane proteins from mammalian, bacterial, yeast, and insect sources.

Section II of the book (chapters 5-8) describes X-ray crystallography as a powerful method for structural determination of membrane proteins. There is a description of various approaches for crystallization of proteins and using these data for understanding fine structure of various membrane proteins.

Section III includes chapters 9-13, where electron microscopic methods are discussed. It includes protocols for electron crystallography, cryoelectron tomography, single particle analysis, and presentation of atomic structures of membrane proteins.

Section IV (chapters 14-18) includes descriptions of nuclear magnetic resonance (NMR) methods of membrane proteins using solution and solid-state NMR. A special chapter highlights NMR spectroscopy of lipid bilayers.

Section V (chapters 19-23) deals with molecular modeling of membrane proteins. There are discussions related to structural models of transmembrane proteins, molecular dynamics of membrane peptides and proteins, and others models.

Besides the evident methodological direction, this book gives a brief introduction into problems related to studies of membrane proteins.

Each chapter contains descriptions of principles of considered methods, lists of chemicals required for use of these methods, sequential steps of these methods, remarks on each step and troubleshooting, and a basic bibliography to each chapter.

I believe that this book will be useful for biochemists, membrane biologists, molecular biologists, and biotechnologists, and also for specialists in cell biology and proteomics. There is no doubt that the material of this book will be very useful for teachers and students of colleges and universities specialized in the fields mentioned above. 